\title{
屏蔽式核主洜水润滑可倾瓦推力轴承推力盘的 离心效应 ${ }^{*}$
}

\author{
宋智翔 1,2 刘莹 1,2 郭飞 1,2 刘向锋 1,2 王玉明 1,2
}

(1. 清华大学摩擦学国家重点实验室 北京 100084;

2. 清华大学机械工程系 北京 100084)

\begin{abstract}
摘要: 屏蔽式核主泵避免了动密封问题, 同时主葲中水润滑可倾瓦推力轴承有着尺寸大、推力盘转动惯量高、离心效应明显 的特点。针对主泵中推力盘的结构特点及工况条件, 基于有限元分析方法, 建立考虑推力盘离心变形的可倾瓦推力轴承多场 耦合计算模型。基于推力盘变形独立性探索了推力盘装配结构中两个关键间隙的设计参数：内轮毫端面与端面板直接接触, 外套环与端面板之间留有间隙, 屏蔽套与外套环之间应至少预留 $1.74 \mathrm{~mm}$ 的间隙; 探讨了推力盘装配结构对离心变形的影响: 低转速下可倾瓦轴承可通过瓦块倾斜补偿推力盘变形, 转速大于 $3600 \mathrm{r} / \mathrm{min}$ 时推力盘变形迅速增大, 极大增加润滑失效可能 性; 分析计算了不同结构尺寸下离心变形对推力轴承工作性能的影响：飞轮内轮毂凸沿外径的合理尺寸范围为 $0.63 \sim 0.67 \mathrm{~m}$ 。
\end{abstract}

关键词: 屏蔽式核主泵; 可倾瓦推力轴承; 离心变形

中图分类号: TH133

\section{Influence of Centrifugal Deformation of Thrust Collar in Water-lubricated Tilting-pad Thrust Bearings of Nuclear Canned Pump}

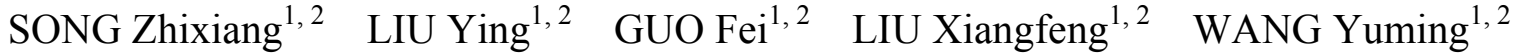

(1. State Key Laboratory of Tribology, Tsinghua University, Beijing 100084;

2. Department of Mechanical Engineering, Tsinghua University, Beijing 100084)

\begin{abstract}
The canned pump avoids the problem of rotating seal. However, the tilting pad thrust bearings in the canned pump have a large size and moment of inertia, which leads to an obvious centrifugal inertia effect. A multi-field coupled numerical model of tilting pad thrust bearings is established based on the infinite element method. Two key values of assembly gaps in flywheel design are discussed. There should be at least $1.74 \mathrm{~mm}$ between the shield and the outer ring. The centrifugal deformations and corresponding performances of tilting pad thrust bearings of different sizes and speed are analyzed. The rotating speed should be controlled within $3600 \mathrm{r} / \mathrm{min}$. Finally, the outer diameter of inner hub of flywheel is discussed and the optimal value ranges from 0.63 to $0.67 \mathrm{~m}$.
\end{abstract}

Key words: canned pump; tilting pad thrust bearing; centrifugal deformation

\section{0 前言}

屏蔽式核主泵是第三代非能动安全原理核电 技术的核心设备 ${ }^{11]}$, 其与轴封式核主泵的最大区别 在于避免了旋转轴的动密封问题。但也带来了新的 技术难点, 即主泵用大尺寸水润滑轴承的设计问 题 ${ }^{[2]}$ 。特别是大尺寸推力轴承工作在高速、重载工 况下, 由于水的低黏度特性, 推力轴承的液膜厚度 会远小于油润滑轴承液膜厚度, 这也使得轴承对轴

* 国家重点基础研究发展资助项目(973 计划, 2015CB057303)。20170718 收到初稿, 20171015 收到修改稿
向变形更加敏感。为增加屏蔽式核主泵转子惰转时 间, 双向推力轴承的推力盘与下部飞轮组合成一体 ${ }^{[3]}$, 从而具有较大转动惯量。这种组合结构的端面 即推力轴承的膜厚边界条件, 端面轴向变形会直接 影响推力轴承的液膜分布, 尤其在高速运行中更加 显著。在低黏度介质润滑中, 可倾瓦推力轴承与推 力盘之间液膜厚度为 $10^{-5} \mathrm{~m}$ 量级, 组合飞轮端面的 轴向变形也可能达到这个量级。

在可倾瓦推力轴承结构的研究中, 学者们对轴 承的支承方式、瓦面设计等问题进行了研究 ${ }^{[4-6]}$ 。为 了满足轴承使用中需要正反转的特性, 可倾瓦轴承 通常采用中心支承的结构, 该结构的油润滑可倾瓦 
轴承, 可以利用热楔效应承载。水润滑介质轴承的 温升不明显, 无法产生足够的承载力 ${ }^{[7]}$ 。在实际使 用中, 通常采用对瓦面形状进行控制, 形成周向凸 表面来增加轴承承载能力, 当凸起高度/最小膜厚在 $0.4 \sim 0.5$ 范围内承载力达到最优 ${ }^{[7]}$ 。

西安交通大学的陈渭、浙江大学的黄滨等对可 倾瓦推力轴承建立了热弹流的润滑模型, 进行了推 力轴承噪声分析、尺寸计算、动特性分析等理论实 验研究, 对可倾瓦轴承在广泛工况参数、介质参数、 几何参数下的性能做了探讨 ${ }^{[8-12]}$ 。之前学者主要集 中关注推力瓦及推力盘在液膜压力及温度不均匀分 布下的变形, 推力盘由离心力产生的轴向变形部分 却极少有学者进行研究。

本文基于有限元方法, 统一建模计算分析不同 尺寸轴承在不同转速下推力盘端面的轴向变形。基 于考虑飞轮组合结构的有限元模型进行了推力盘端 面变形独立性分析, 在此基础上将端面变形结果代 入可倾瓦推力轴承的多场耦合性能计算中, 为大型 水润滑推力轴承理论分析模型的进一步完善及轴承 运行规律研究提供参考。

\section{1 推力轴承计算模型}

\section{1 几何模型}

屏蔽式核主百中双向水润滑可倾瓦推力轴承 采用中心支承形式。为满足中心支承下可倾瓦轴承 承载力需求, 在可倾瓦轴承表面沿周向预加工有一 定高度的表面圆弧形凸起(图 1)。

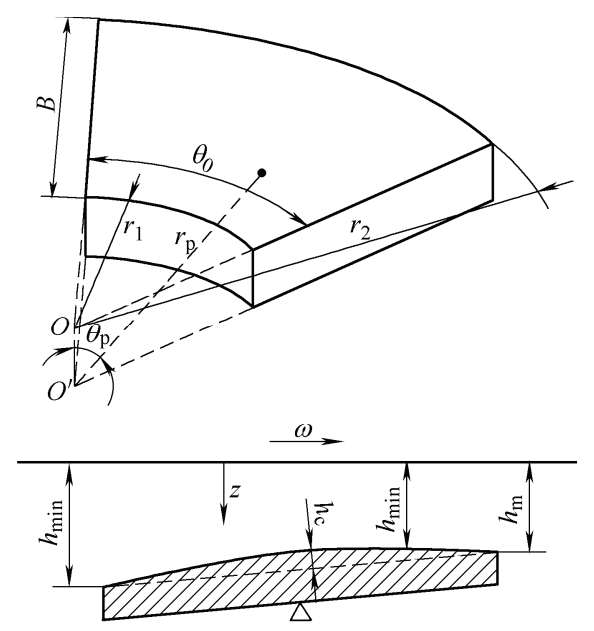

图1几何模型示意图

可倾瓦推力轴承的轴瓦为周向均布, 具有对称 结构, 在建立几何模型时, 为减小模型计算量, 提 高计算效率, 根据研究对象特点进行模型简化 ${ }^{[13]}$ 。 本文选择单瓦进行建模分析。这种简化基于主轴在
没有角向偏差前提下，瓦块膜厚分布一致，因此可 以通过单瓦润滑性能来评价推力轴承的性能。

图 1 中, $\omega$ 为轴承旋转速度, $h_{\min }$ 为可倾瓦推 力轴承最小膜厚值, $h_{\max }$ 为轴承最大膜厚值, $B$ 是 轴承宽度, $\theta_{0}$ 为轴承包角, 轴承内外半径分别为 $r_{1}$ 与 $r_{2}$, 分析采用柱坐标系, $r$ 为径向坐标, $\theta$ 为周向 坐标, $z$ 为膜厚方向坐标。

整瓦膜厚分布函数 $h(r, \theta)$ 表达方程为

$$
\begin{gathered}
h=\sin \gamma_{p}\left[r \sin \left(\theta_{p}-\theta\right)-r_{m} \sin \left(\theta_{p}-\theta_{m}\right)\right]+ \\
h_{m}+h_{c}(\theta)+\Delta h
\end{gathered}
$$

式中, $h_{\mathrm{m}}$ 为不计入弧形凸表面时最小膜厚值; $\theta_{\mathrm{p}}$ 为 节线位置角; $\gamma_{\mathrm{p}}$ 为瓦块绕节线转过的倾斜角; $r_{\mathrm{m}} 、 \theta_{\mathrm{m}}$ 分别为不计入弧形凸表面时最小膜厚处的径向坐标 及周向坐标; $h_{\mathrm{c}}$ 为弧形凸表面带来的膜厚修正量; $\Delta h$ 为推力盘轴向变形带来的膜厚修正量。 $h_{\text {min }}$ 为考 虑凸表面及推力盘变形后的最小膜厚。

\section{2 物理模型}

可倾瓦推力轴承的物理计算模型中, 主要考虑 推力盘与推力瓦块之间液膜流态、流场分布及温度 场分布及其耦合作用。其中流体压力分布的控制方 程为考虑流态的雷诺方程

$$
\frac{\partial}{\partial r}\left(\frac{r h^{3}}{k_{r} \mu} \frac{\partial p}{\partial r}\right)+\frac{\partial}{r \partial \theta}\left(\frac{h^{3}}{k_{\theta} \mu} \frac{\partial p}{\partial \theta}\right)=\frac{r \omega}{2} \frac{\partial h}{\partial \theta}
$$

式中, $p$ 为每点处压强; $\mu$ 为润滑介质动力黏度; $\omega$ 为轴承转速; $k_{\mathrm{r}} 、 k_{\theta}$ 为与流态相关的紊流系数。目前 对于紊流现象的描述模型主要有 Ng-Pan 模型 ${ }^{[14]}$ 、 青木弘模型 ${ }^{[15]}$ 、Constantinescu 模型 ${ }^{[16]}$ 及 Hirs 模 型 ${ }^{[17]}$, 这些模型都有各自不同的适用范围。此处采 用 Ng-Pan 的紊流模型, $k_{\mathrm{r}} 、 k_{\theta}$ 可表达为雷诺数 $R e$ 的 表达式。

$$
\begin{aligned}
& \operatorname{Re}<1500 \text { 时 } \\
& \qquad k_{r}=k_{\theta}=12 \\
& \operatorname{Re}>1500 \text { 时 } \\
& k_{r}=12+0.0136 R e^{0.9} \\
& k_{\theta}=12+0.0043 R e^{0.96}
\end{aligned}
$$

流场求解中压力边界条件为雷诺边界条件, 在 内外径处及入口边和出口边

$$
\begin{array}{ll}
\left.p\right|_{r=r_{i}}=0 & \left.p\right|_{r=r_{o}}=0 \\
\left.p\right|_{\theta=\theta_{i}}=0 & \left.p\right|_{\theta=\theta_{o}}=0
\end{array}
$$

当计算压力值为负值时, 此处发生液膜破裂, 此时的压力边界为 


$$
\frac{\partial P}{\partial r}\left|\Gamma_{r}=0 \quad \frac{\partial P}{\partial \theta}\right| \Gamma_{\theta}=0
$$

式中, $\Gamma_{r} 、 \Gamma_{\theta}$ 为液膜破裂的径向、周向边界位置。 流体温度场控制方程为能量方程

$$
\begin{aligned}
& \rho C_{p}\left[\left(6 \omega r h-\frac{h^{3}}{\mu} \frac{\partial p}{r \partial \theta}\right) \frac{\partial t}{r \partial \theta}-\frac{h^{3}}{\mu} \frac{\partial p}{\partial r} \frac{\partial t}{\partial r}\right]= \\
& \frac{12 \mu \omega^{2} r^{2}}{h}+\frac{h^{3}}{\mu}\left[\left(\frac{\partial p}{r \partial \theta}\right)^{2}+\left(\frac{\partial p}{\partial r}\right)^{2}\right]+12 \lambda \tau
\end{aligned}
$$

式中, $\rho$ 为润滑介质密度; $C_{\mathrm{p}}$ 为润滑介质比热容; $t$ 为液膜温度。热边界条件为

$$
\left.t\right|_{\theta=\theta_{0}}=t_{\text {in }}
$$

此处的入口温度 $t_{i n}$ 考虑水介质的冷热混合, 即 认为自上一块扇形瓦中出口边流出的水介质与循环 供水混合后进入下一块扇形瓦中, 混合后的入口温 度可以根据下式计算出 ${ }^{[18]}$

$$
t_{\text {in }}=\frac{t_{r e c} Q_{r e c}+t_{\text {sup }} Q_{\text {sup }}}{Q_{\text {rec }}+Q_{\text {sup }}}
$$

式中, $t_{r e c}$ 为上一块扇形瓦中流出的热流体温度; $t_{\text {sup }}$ 为补充的循环供水温度; $Q_{r e c}$ 为上一块扇形瓦出口 边流量; $Q_{\text {sup }}$ 为供液流量。

此模型还考虑水的黏温特性, 介质黏温方程采 用 Vogel 方程

$$
\mu=\exp \left(A+\frac{B}{t+C}\right)
$$

式中, $A 、 B 、 C$ 为与介质相关的系数, $t$ 为温度值。

\section{2 简化圆盘的离心变形}

为确认推力盘在离心力作用下轴向变形量的 大小, 首先对推力盘的离心变形进行简化分析。将 推力盘假设为实心均匀圆环, 在有限元软件中以一 定速度旋转, 建模计算离心力对端面轴向位移的 影响。

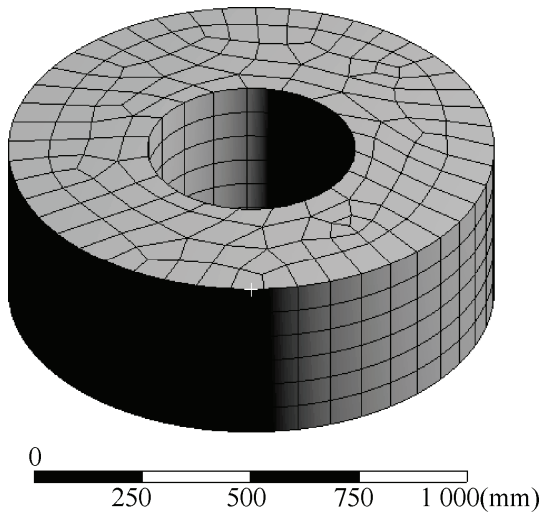

图 2 圆盘模型网格划分
在 ANSYS Workbench 中对圆环形飞轮建立了 简单仿真模型, 计算模型的网格划分及边界条件如 图 2 3 所示。因为此模型为量级分析, 故使用软件 默认精度网格进行求解。图 3 中 $S_{1}$ 为整体绕其轴线 的角速度, 用来模拟推力盘旋转时离心力作用, $S_{2}$ 为施加在底面外径处的轴向位移约束, 限制圆盘整 体刚性位移。此外，由于屏蔽主泵为立式结构，对 整个圆盘施加铅垂向下(图中 $-Y$ 方向)整体重力加 速度。

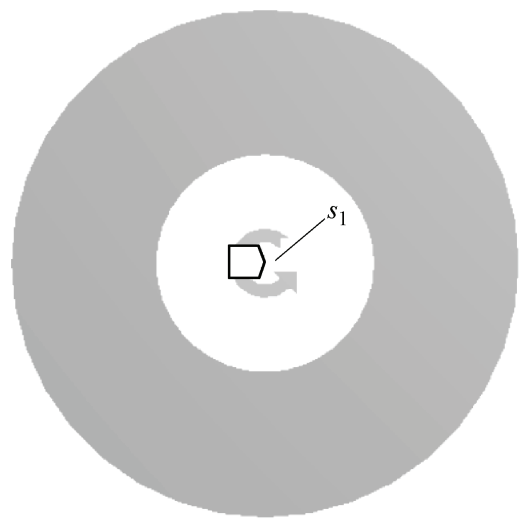

整体角速度 $188.5 \mathrm{rad} / \mathrm{s}$

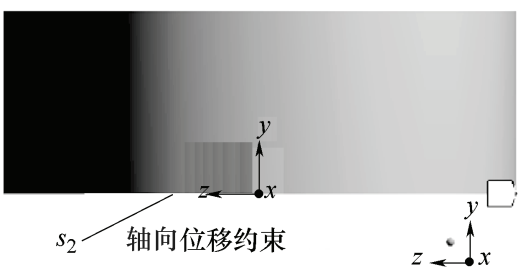

图 3 圆盘有限元模型的载荷及约束

基于上述有限元仿真模型计算不同尺寸、不同 工况下推力盘轴向变形。相关输入参数如表 1 所示。

\section{表 1 圆盘模型输入参数}

\begin{tabular}{cc}
\hline \multicolumn{1}{c}{ 参数 } & 取值 \\
\hline 推力盘外径 $D_{\mathrm{o}} / \mathrm{mm}$ & $280 \sim 1560$ \\
推力盘内外径比 $D_{\mathrm{i}} / D_{\mathrm{o}}$ & $3: 7$ \\
飞轮厚度 $h / \mathrm{mm}$ & 350 \\
转速 $n /(\mathrm{r} / \mathrm{min})$ & $300 \sim 3000$ \\
\hline
\end{tabular}

上端面变形结果如图 4 所示, 由离心力产生推 力盘轴向变形关于转轴是轴对称分布, 变形后端面 近似为锥面。故只需要端面内外径处变形量 $\Delta h_{1}$ 及 $\Delta h_{2}$ 即可表征离心力带来的轴向变形分布。

端面变形分布量

$$
\Delta h=\Delta h_{1}+\left(\Delta h_{2}-\Delta h_{1}\right)\left(r-r_{1}\right) /\left(r_{2}-r_{1}\right)
$$

在不同尺寸及转速下, 推力盘端面轴向离心变 形量如图 5 所示。从图 5 中可以看到，不同尺寸下 推力盘离心力产生的轴向变形量差异较大。大尺寸 下尤其是在转速较大时轴向变形量较为明显。离心 
力 $F=m r \omega^{2}$, 从计算中也可以看到, 考虑到水润滑 推力轴承正常工况下最小膜厚为 $10^{-5} \mathrm{~m}$ 量级, 因 此当推力轴承外径小于 $560 \mathrm{~mm}$ 时基本可以忽略推 力盘离心力带来的影响。大型水润滑可倾瓦推力轴 承外径一般在 $1000 \mathrm{~mm}$ 左右, 其离心效应不应忽 略, 当转速 $n>1000 \mathrm{r} / \mathrm{min}$ 时离心力带来的推力盘 轴向变形尤其严重, 此时已经不可忽略推力盘变形。

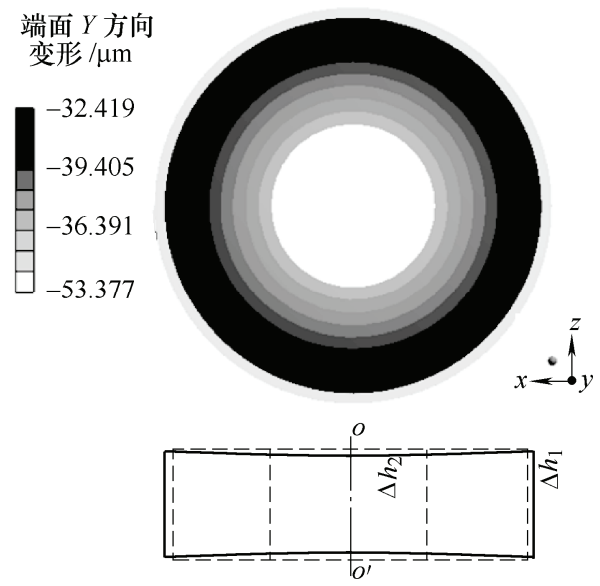

图 4 $1800 \mathrm{r} / \mathrm{min}$ 时圆盘模型端面轴向变形

\section{3 考虑装配结构的有限元分析}

\section{1 有限元分析模型}

经过圆盘模型分析, 推力盘离心变形量与水膜 厚度为同一量级, 不可忽略。由于屏蔽主洜中飞轮 组件有较为复杂的装配结构, 这些装配关系对端面 轴向变形可能产生影响。

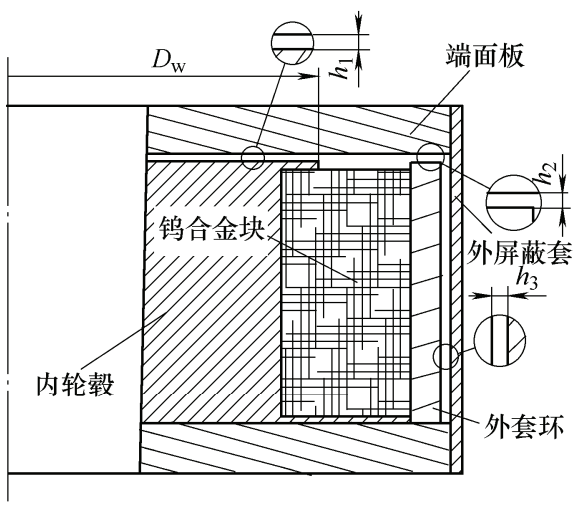

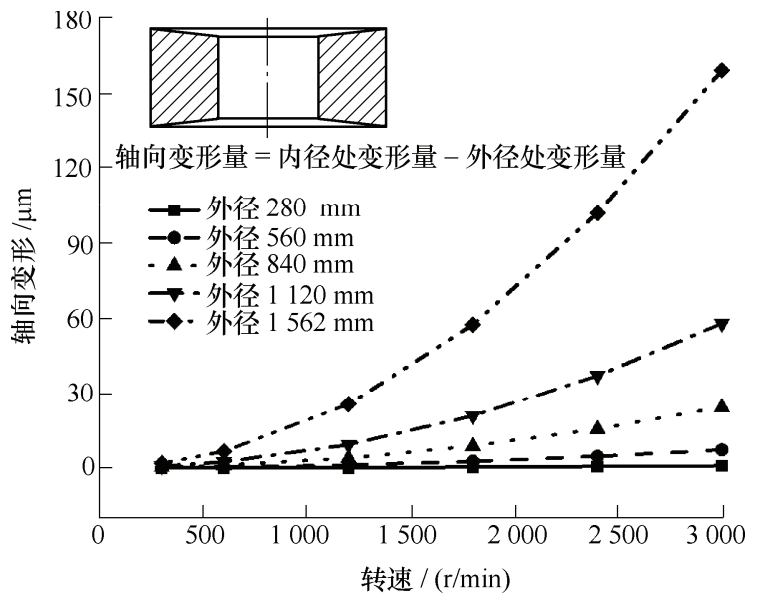

图 5 不同尺寸下离心变形结果

针对屏蔽主泵中推力盘组合结构, 在 ANSYS APDL 中进一步进行建模计算。同样地，根据其结 构特点, 计算采用 $1 / 12$ 对称计算模型, 即一个顶角 为 $30^{\circ}$ 的扇形区域，减少建模及计算工作量。模型 中铇合金块材料为铇合金，其余部分按高强度不锈 钢计算。

计算模型相关主要参数如表 2 所示。几何计算 模型如图 6 所示。

\section{表 2 有限元分析模型输入参数}

\begin{tabular}{cc}
\hline 参数 & 取值 \\
\hline 推力盘外径 $D_{\mathrm{o}} / \mathrm{mm}$ & 1000 \\
推力盘内外径比 $D_{\mathrm{i}} / D_{\mathrm{o}}$ & $3: 7$ \\
飞轮厚度 $h / \mathrm{mm}$ & 380 \\
转速 $n /(\mathrm{r} / \mathrm{min})$ & $600 \sim 3600$ \\
\hline
\end{tabular}

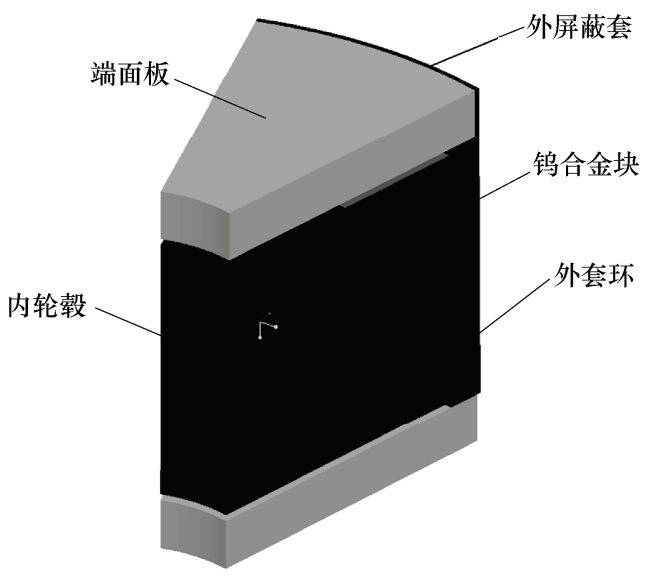

图 6 ANSYS 装配计算几何模型

此计算模型中, 飞轮主要由端面板(即轴承推力 面)、内轮㙪、铇合金块、外套环及外屏蔽套这几部 分组成。飞轮装配部件及装配位置关系中, 主要存 在 $h_{1} 、 h_{2} 、 h_{3}$ 这三个设计参量, 分别表示内轮毂与 端面板、端面板与外套环、屏蔽套与外套环之间的
配合关系。为保证端面板的轴向定位位置, $h_{1} 、 h_{2}$ 其中一个值必须为 0 , 后续将分成两种情况分别讨 论。外屏蔽套与端面板之间作焊接处理。

有限元分析所施加的边界条件如图 7 所示, 在 端面施加液膜压力载荷, 整体添加绕轴心的角速度, 
从而得到最接近实际工况中的端面变形情况。

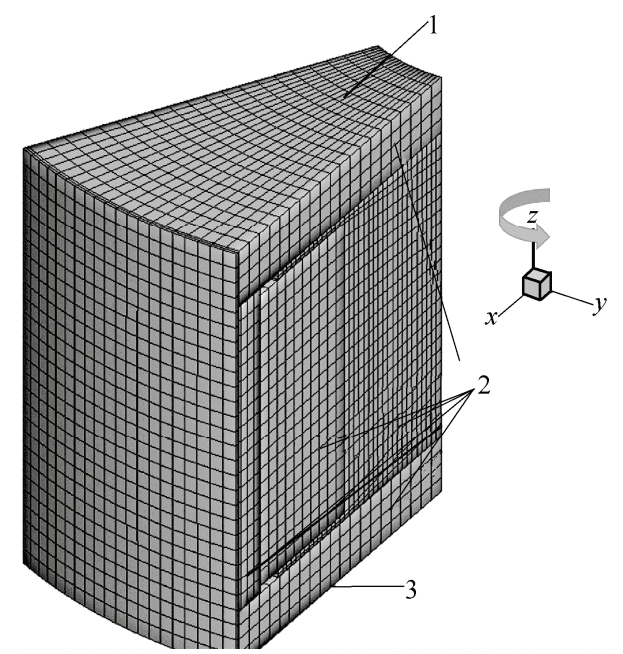

图 7 ANSYS 装配计算的网格划分及边界条件

1、3. 表面压力分布 2 . 周期性边界条件, 角速度 $\Omega$

\section{2 推力盘变形独立性分析}

在飞轮与端面板装配结构设计中, 一个主导设 计思想应为尽量避免飞轮组件与端面板之间的变形 产生耦合, 保证端面板轴向变形独立性。下面对装 配关系中的间隙设计分别进行讨论。

假设端面板通过屏蔽套实现轴向定位, 即 $h_{2}=$ 0 。屏蔽套与外套环、端面板与外套环之间没有变形
协调问题，具有变形独立性(此时 $h_{1}>0, h_{3}>0$ )。 首先单独对内轮㪍、钨合金块及外套环进行结构建 模，模型中外套环与铇合金块之间为过盈配合。分 析在不同转速下三者组合结构的变形关系。

从图 8 中可以看到, 外套环径向位移约为 1.74 $\mathrm{mm}$, 屏蔽套径向位移最小处仅有 $7 \mu \mathrm{m}$ 。如果要避 免外套环对屏蔽套的变形产生影响, 二者间隙 $h_{3}$ 需 要大于 $1.74 \mathrm{~mm}$ 。从图 8 中可以看到, 正常工况下 外套环轴向变形为向内收缩, 内缩位移 $143 \mu \mathrm{m}$, 端 面板轴向内缩位移约为 $80 \mu \mathrm{m}$, 小于外套环的轴向 位移。初始静止条件下设置二者间隙 $h_{2}=0$, 在离 心力作用下产生了轴向间隙, 即外套环无法始终支 承端面板且不会对端面板的轴向位移产生影响, 所 以理论上不能采用外套环对端面板实现轴向定位。

其次考虑内轮勃的影响, 从图 $8 \mathrm{e}$ 中可以看到, 此时端面板内径处的轴向位移最大可达到 500 $\mu \mathrm{m}$ (向内凹), 比正常工况下可倾瓦推力轴承的润滑 膜最小厚度 (约为 $10^{-5} \mathrm{~m}$ 量级)大一个量级。从图 $8 \mathrm{c}$ 中可以看到, 内轮鈠端面的轴向位移约为 $10 \mu \mathrm{m}$ (向 外延展), 故飞轮组合结构中, 内轮毂与端面板轴向 接触, 即间隙 $h_{1}=0$, 理论上端面板依靠内轮毂实现 轴向定位。

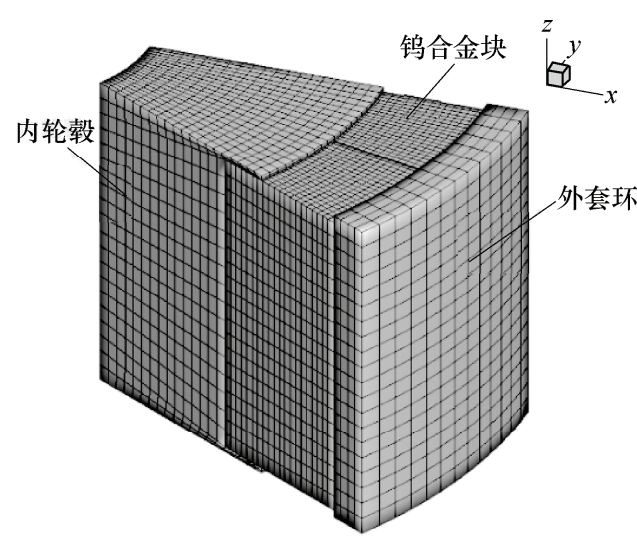

(a) 内部三体组件模型

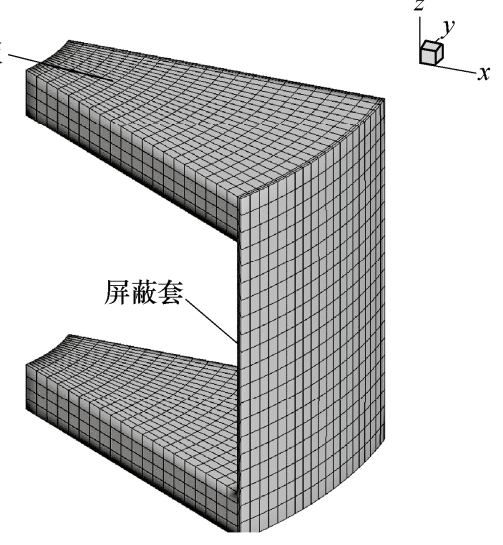

(b) 外部二体组件模型

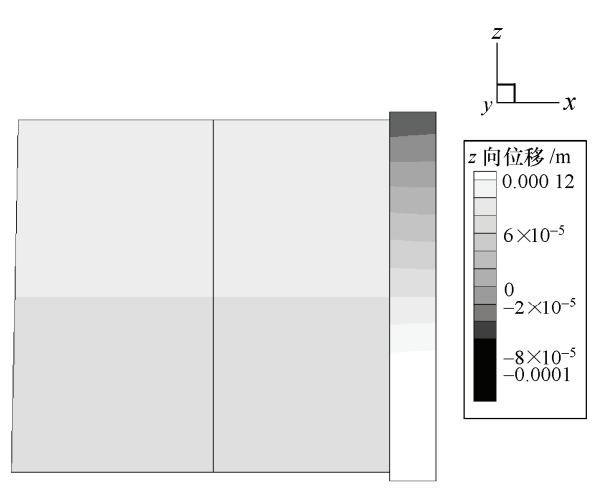

(c) 三体组件的轴向位移分布

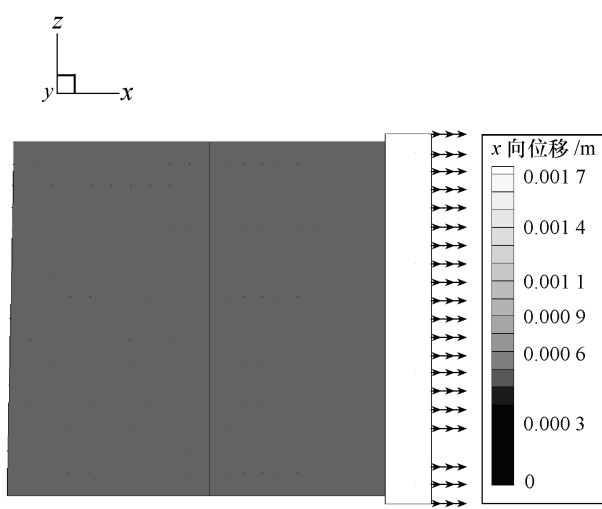

(d) 三体组件的径向位移分布 


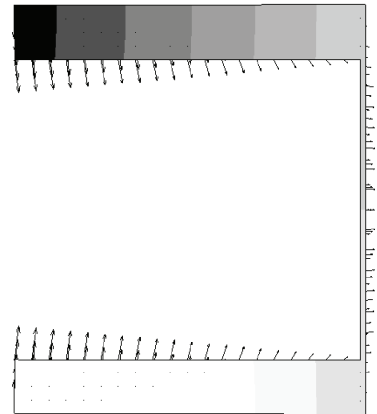

(e)二体组件的轴向位移分布
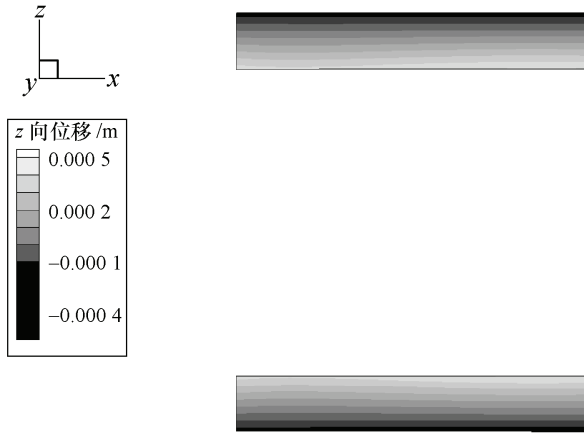

(f) 二体组件的径向位移分布

图 8 内轮㷤、铇合金块、外套环三体模型, 外套环、屏蔽套二体模型及计算结果 $(1800 \mathrm{r} / \mathrm{min})$

建立内轮趾表面与端面板接触 $\left(h_{1}=0\right)$ 的飞轮完 整结构装配模型进行分析(图 9)。从图 9 中可以看 到, 端面板最大相对变形量(最大值与最小值之差) 为 $16 \mu \mathrm{m}$, 与液膜膜厚为同一量级, 因此, 由于离 心效应产生的轴向变形在性能分析中会有影响。本 文后续均基于内轮誖表面与端面板接触 $\left(h_{1}=0\right)$ 完整 装配结构模型进行分析。

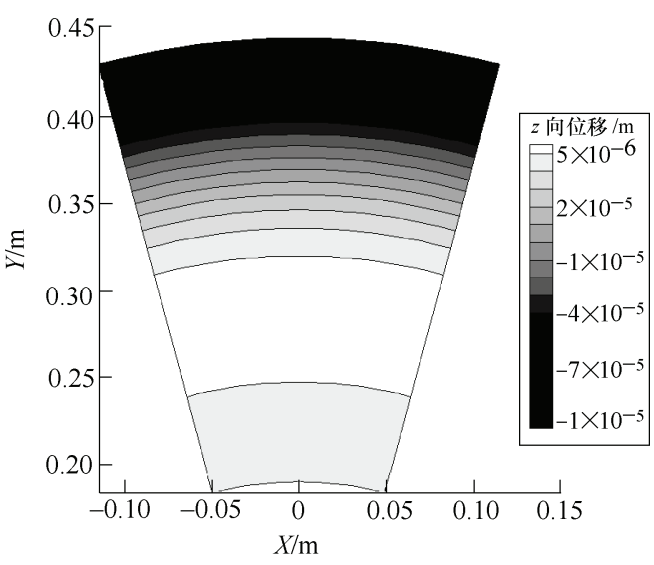

图 9 完整装配结构下端面变形(1 $800 \mathrm{r} / \mathrm{min})$

\section{3 不同转速下轴向变形探究}

从图 10 中可以看到, 随着转速的增大, 推力 盘端面轴向变形的形状也在改变, 且变形的量级与 水润滑推力轴承的最小膜厚可相比拟, 此时推力盘
的离心力的影响不可忽略。当转速大于 $3600 \mathrm{r} / \mathrm{min}$ 时，推力盘的变形显著增大。

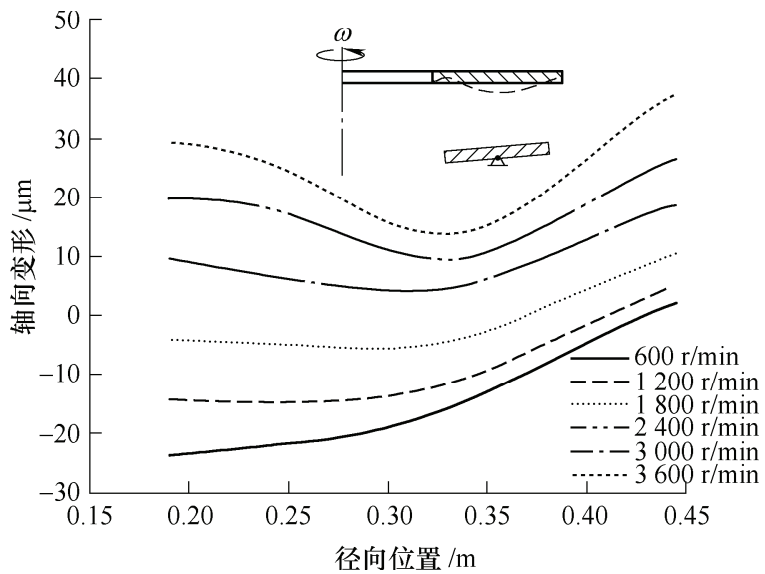

图 10 不同转速下推力盘端面的离心变形

为了详细考察离心力对轴承性能的影响, 可以 得到同等工作载荷、不同转速下考虑推力盘离心力 后的膜厚分布(图 11)。结果表明，在高转速下，推 力盘变形十分严重, 同时推力轴承碰磨的机会也大 大增加。离心变形计算后再将端面变形量提取代入 可倾瓦推力轴承的计算程序中进行多场耦合计算。 不同转速下离心力对推力轴承性能的影响如图 12 所示。计算输入参数如表 3 所示。
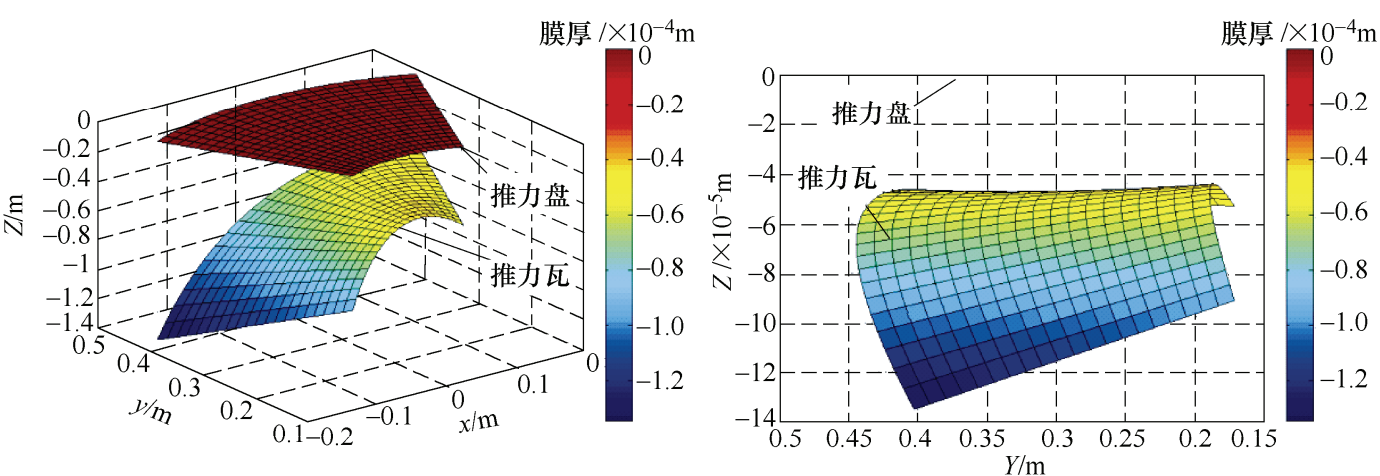

(a) $1800 \mathrm{r} / \mathrm{min}$ ，不考虑离心变形 

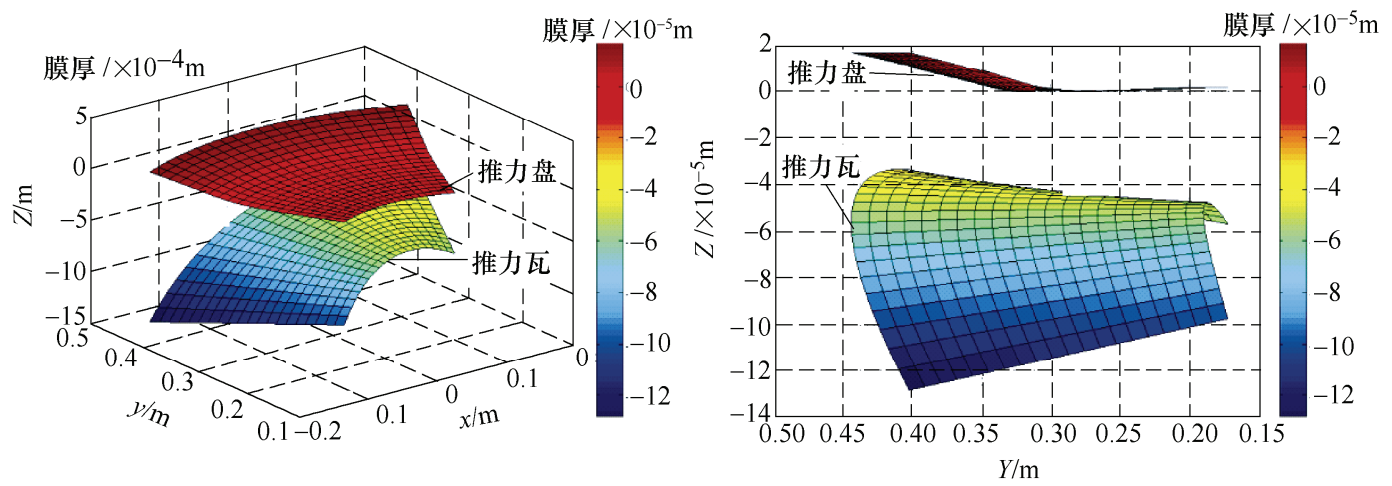

(b) $1800 \mathrm{r} / \mathrm{min}$, 考虑离心变形
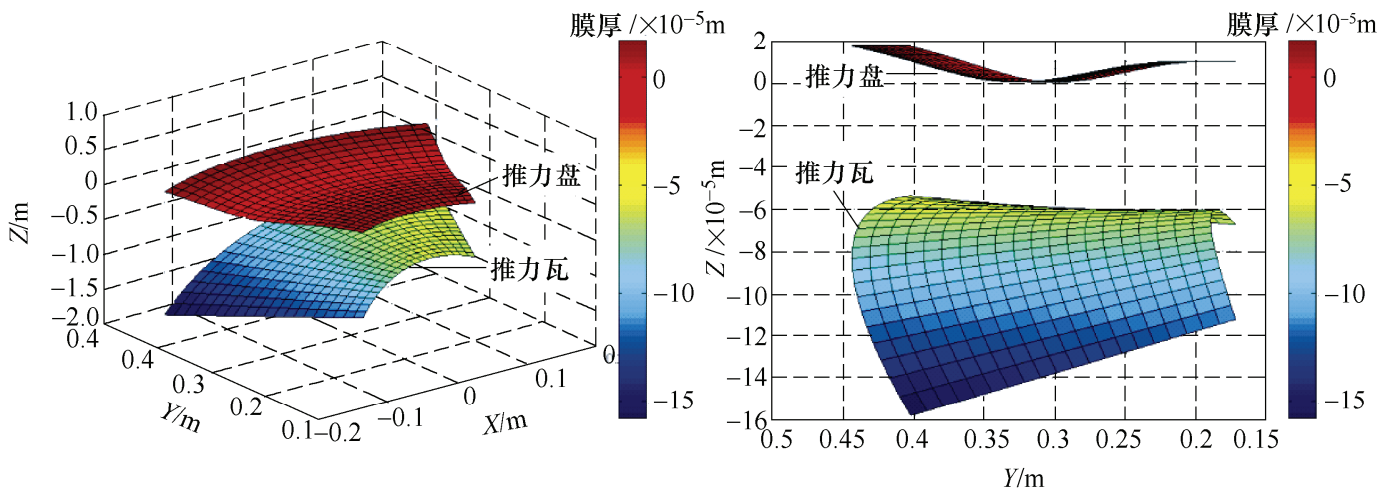

(c) $3000 \mathrm{r} / \mathrm{min}$, 考虑离心变形

图 11 不同转速下的膜厚分布

\section{表 3 理论模型输入参数}

\begin{tabular}{lc}
\hline \multicolumn{1}{c}{ 参数 } & 取值 \\
\hline 瓦块内径 $r_{\mathrm{i}} / \mathrm{mm}$ & 455.5 \\
瓦宽 $B / \mathrm{mm}$ & 254 \\
包角 $\theta_{0} /\left(^{\circ}\right)$ & 50 \\
径向支点偏心系数 & 0.54 \\
周向支点偏心系数 & 0.5 \\
瓦块表面凸起高度 $h_{\mathrm{c}} / \mu \mathrm{m}$ & 25 \\
转速 $n /(\mathrm{r} / \mathrm{min})$ & $600 \sim 6000$ \\
单瓦载荷 $W / \mathrm{kN}$ & 22 \\
入口水温 $/{ }^{\circ} \mathrm{C}$ & 65 \\
\hline
\end{tabular}

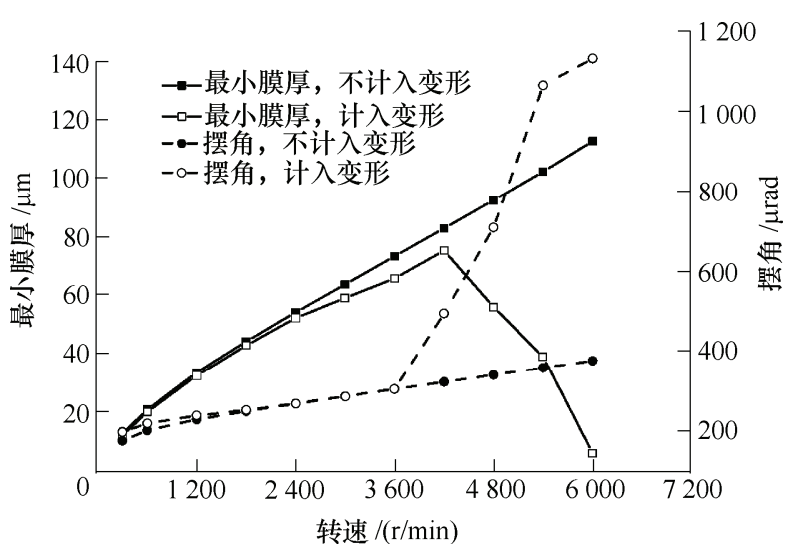

图 12 不同转速下离心效应的影响

载荷相同时, 轴承最小膜厚反映其承载能力。 从图 12 中可以看到, 转速 $n<3600 \mathrm{r} / \mathrm{min}$ 时, 随着
转速的增大, 轴承的最小膜厚及摆角 $\gamma_{\mathrm{p}}$ 随之增大, 计入推力盘离心变形后结果与未计入结果相近, 即 此转速范围内推力盘离心变形对轴承润滑性能影响 不大。轴承的性能参数中, 离心变形带来的最显著 的影响是对内侧泄漏量的影响, 最大的相对影响可 以达到 9.4\%, 这是因为推力盘的离心变形增大了内 径处的膜厚，使得泄漏量增大。

转速增大到 $3600 \mathrm{r} / \mathrm{min}$ 以上时, 由于离心变形 的进一步增大, 瓦块倾摆已经无法补偿离心变形(图 11), 轴承性能受到剧烈影响, 最小膜厚很快减小, 摆角急速增大, 轴承丧失大部分承载能力, 发生碰 磨的可能性大大增加, 使用中应避免大尺寸推力轴 承在高转速(大于 $3600 \mathrm{r} / \mathrm{min}$ )下运行。

\section{4 飞轮结构参数的影响}

飞轮组件中内轮晸凸沿的外径尺寸有利于铇 合金块的轴向安装定位, 但由于它与端面板直接接 触, 会影响端面板的离心应变, 所以进一步分析了 内轮钤外径尺寸对离心变形的影响。设铇合金块的 内径为 $0.58 \mathrm{~m}$, 外径为 $0.85 \mathrm{~mm}$, 内轮毂的凸沿外 径 $D_{\mathrm{w}}$ 分别取 $0.63 \mathrm{~m} 、 0.67 \mathrm{~m} 、 0.71 \mathrm{~m} 、 0.75 \mathrm{~m} 、 0.79$ $\mathrm{m}$ 。选取 $1800 \mathrm{r} / \mathrm{min}$ 的工况计算, 飞轮组件的轴向 变形如图 13 所示。

相应地, 在不同的尺寸参数下, 考虑推力盘的 离心变形, 可倾瓦推力轴承的润滑性能由 Matlab 联 
合计算得到, 如图 14 所示。

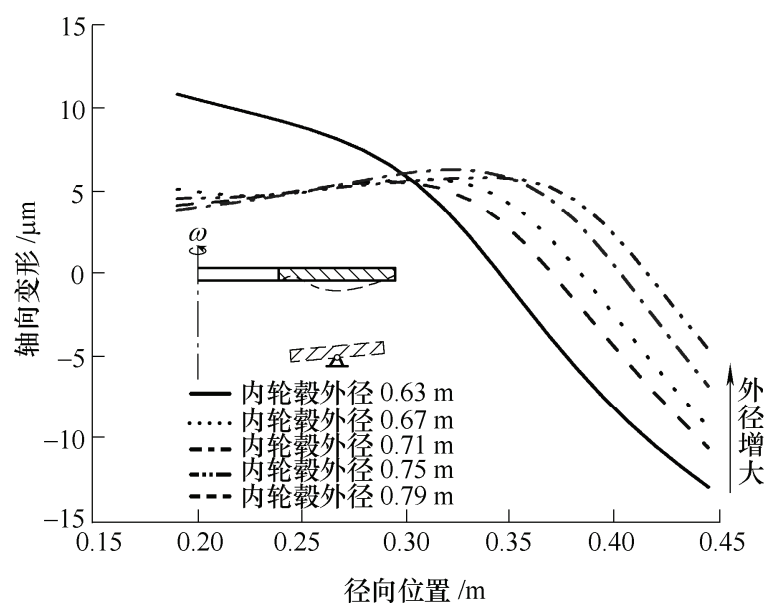

图 13 内轮珲外径对离心变形的影响

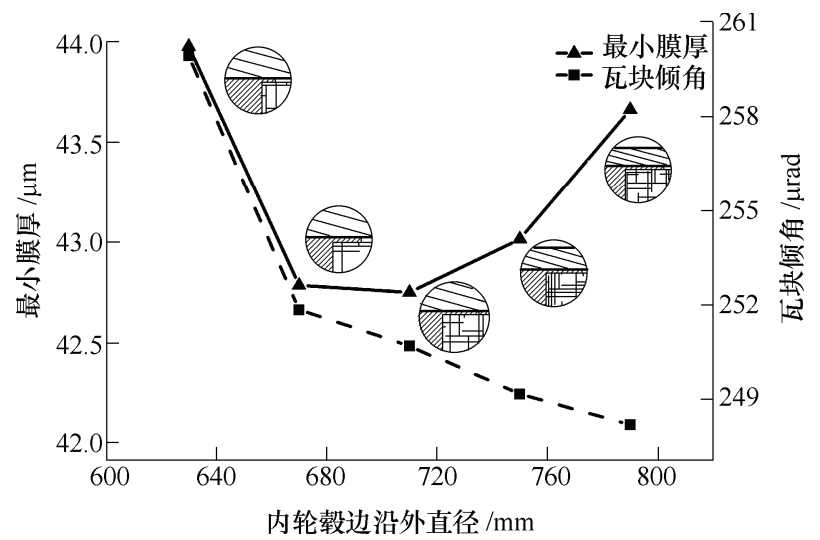

图 14 内轮毂外径对轴承性能的影响

从图中可以看到, 推力盘的结构尺寸对于组件 结构在高速下的离心变形有直接影响, 从而对推力 轴承的润滑性能造成影响。在相同载荷下, 最小膜 厚越大, 其承载能力越强; 瓦块倾角越大, 越容易 造成瓦块的碰磨。从计算结果中可以看到, 内轮勃 外径 $D_{w}>0.67 \mathrm{~m}$ 时推力盘的离心变形趋于一致, 同 时考虑瓦块最小膜厚与倾角, 以及内轮毂加工的工 艺性, 内轮毂外径应选取 $0.63 \sim 0.67 \mathrm{~m}$ 范围内。

\section{4 结论}

（1）使用简化圆盘模型分析了不同尺寸及工况 下推力盘离心力造成的轴向变形的大小, 发现外径 $D_{\mathrm{o}}>560 \mathrm{~mm}$ 的推力轴承在转速 $n>1000 \mathrm{r} / \mathrm{min}$ 时推 力盘的离心变形与水膜为同一量级, 不可忽略。

(2) 针对屏蔽式核主泵中飞轮与推力盘端面板 的组合结构进行了有限元分析, 针对组合结构中的 关键间隙参数进行了讨论分析。基于推力盘变形独 立性提出了相关参数的取值范围: $h_{1}=0$, 内轮毂端 面与端面板直接接触; $h_{2}>0$, 即外套环与端面板之
间留有间隙; $h_{3}$ 处屏蔽套与外套环之间应至少预留 $1.74 \mathrm{~mm}$ 的间隙。

（3）基于有限元结构分析, 发现随着转速的增 大, 主泵中推力轴承推力盘的轴向变形会显著增大, 直接影响可倾瓦轴承润滑性能。低转速下可倾瓦轴 承可以通过瓦块倾斜补偿推力盘变形, 转速 $n>$ $3600 \mathrm{r} / \mathrm{min}$ 时, 推力盘变形迅速增大, 极大增加润 滑失效可能性，实际工作中应避免这种工况。

（4）根据计算结果, 给出屏蔽式核主百中内轮 瑴凸沿外径 $D_{\mathrm{w}}$ 在考虑组件离心变形时的合理尺寸 范围是 $0.63 \sim 0.67 \mathrm{~m}$ 。

\section{参 考 文 献}

[1] 孙汉虹. 第三代核电技术 AP1000[M]. 北京: 中国电力 出版社, 2010.

SUN Hanhong. The third generation nuclear power technology[M]. Beijing: China Power Press, 2010.

[2] 欧阳武, 张帆, 王建否, 等. 低黏润滑轴承可靠性强化 试验中磁力加载研究[J]. 机械工程学报, 2015, 51(4): 199-205.

OUYANG $\mathrm{Wu}$, ZHANG Fan, WANG Jianlei, et al. Research on magnetic loading in reliability enhancement testing of low viscosity lubricated bearings[J]. Journal of Mechanical Engineering, 2015, 51(4): 199-205.

[3] 金飞. 核电站屏蔽主泵推力轴承运行与维护探讨 [J]. 中国高新技术企业，2015(13): 137-139.

JIN Fei. Discussion on the operation and maintenance of the shield main pump thrust bearing in the nuclear power station[J]. China High-Tech Enterprises, 2015(13) : 137-139.

[4] 黄滨, 吴军令, 武中德, 等. 双向推力轴承支承结构对 润滑性能的影响[J]. 排灌机械工程学报, 2012, 30(6): 690-694.

HUANG Bin, WU Junling, WU Zhongde, et al. Effects of support structure on lubricating properties of bi-directional thrust bearings[J]. Journal of Drainage and Irrigation Machinery Engineering, 2012, 30(6): 690-694.

[5] 蒋秀龙, 方静辉, 汪久根. 入口坡形对推力滑动轴承润 滑性能的影响 [J]. 润滑与密封, 2010, 35(6): 24-28.

JIANG Xiulong, FANG Jinghui, WANG Jiugen. Effects of Inlet ramps on thermo hydrodynamic lubrication of thrust bearing[J]. Lubrication Engineering, 2010, 35(6): 24-28.

[6] YUAN Xiaoyang, ZHU Jun, CHEN Zhilann, et al. A three-dimensional TEHD model and an optimum surface 
profile design of pivoted pad thrust bearings with large dimensions[J]. Tribology Transactions, 2003, 46(2): 153-160.

[7] 黄成铭. 反应堆主䡕水润滑推力轴承的设计研究 [J]. 机械, 1990(3): 4-11.

HUANG Chengming. The design and research of the water-lubricated thrust bearings in the main reactor pump[J]. Machinery, 1990(3): 4-11.

[8] 陈渭, 朱均. 大型推力轴承热弹流动力润滑的性能计算 [J]. 机械科学与技术, 1994(4): 51-55.

CHEN Wei, ZHU Jun. Performance of large thrust bearing thermal elastohydrodynamic lubrication[J]. Mechanical Science and Technology for Aerospace Engineering, 1994(4): 51-55.

[9] 蒋秀龙. 可倾瓦推力滑动轴承弹流润滑研究[D]. 杭州: 浙江大学, 2011.

JIANG Xiulong. TEHD analysis of tilting pad thrust beaing[D]. Hangzhou: Zhejiang University, 2011.

[10] 董胜先, 马求山. 大型重载推力轴承热弹流计算及尺寸 效应 $[\mathrm{J}]$. 润滑与密封, 2014(8)：108-111.

DONG Shenxian, MA Qiushan. Thermoelastohydrodynamics analysis and size effect of large heavy thrust bearing[J]. Lubrication Engineering, 2014(8): 108-111.

[11] 何加猛, 王小静, 祁高安, 等. 计入三维热效应对可倾 瓦推力轴承动力特性的影响 $[\mathrm{J}]$. 上海大学学报, 2012, 18(5): 519-524.

HE Jiameng, WANG Xiaojing, QI Gaoan, et al. Influence of considering 3D thermal effects on dynamic characteristics of tilting pad thrust bearing $[\mathrm{J}]$. Journal of Shanghai University, 2012, 18(5): 519-524.

[12] 武中德, 张宏, 任忠海, 等. 小支柱簇双层瓦推力轴承 热弹流润滑性能分析 $[\mathrm{J}]$. 哈尔滨工业大学学报, 2003, 35(1): 81-84.

WU Zhongde, ZHANG Hong, REN Zhonghai, et al. Performance analysis for thermo-elastic-hydrodynamic lubrication of thrust bearing with pins and double layer system[J]. Journal of Harbin Institute of Technology, 2003, 35(1): 81-84.

[13］曾攀. 基于 ANSYS 平台有限元分析手册 [M]. 北京: 机 械工业出版社, 2011.

ZENG Pan. Finite element analysis guide: Modeling and analysis of structure[M]. Beijing: China Machine Press, 2011.

[14] CONSTANTINESCU V. Analysis of bearings operating in turbulent regime[J]. Journal of Fluids Engineering, 1962, 84(1): 139-151.

[15] PAN C. A linearized turbulent lubrication theory[J]. Journal of Fluids Engineering, 1965: 675-688.

[16] 原田正躬, 青木弘. スラストす心゙り軸受の乱流潤滑理 論[C]// 日本機械学会論文集 C 編, 1987, 53(496): 2655-2663.

HARADA M, HIROSHI A. Turbulent lubrication theory of sliding thrust bearing[C]// JSME International Part C, 1987, 53(496): 2655-2663.

[17] HIRS G. A bulk-flow theory for turbulence in lubricant films[J]. Journal of Tribology, 1973, 95(2): 137-145.

[18] 张振山. 计入非牛顿、变形及表面形貌效应的动载轴承 热流体动力润滑分析 [D]. 上海: 上海交通大学, 2014 . ZHANG Zhenshan. Analysis of thermohydrodynamic performance of dynamically loaded bearings in consideration of non-netonian, deformation and surface topograhpy[D]. Shanghai : Shanghai Jiao Tong University, 2014.

作者简介: 宋智翔, 男, 1992 年出生, 博士研究生。主要研究方向为流 体润滑。

E-mail: songzx13@mails.tsinghua.edu.cn

刘向锋(通信作者), 男, 1961 年出生, 博士, 教授, 博士研究生导师。 主要研究方向为机械设计, 摩擦学。

E-mail: liuxf@tsinghua.edu.cn 